\title{
Tissue-dependent and -independent gene expression changes in metastatic colon cancer
}

\author{
WILLIAM H. GMEINER ${ }^{1}$, GARY M. HELLMANN ${ }^{2}$ and PERRY SHEN ${ }^{3}$ \\ ${ }^{1}$ Department of Cancer Biology, Wake Forest University School of Medicine, Winston-Salem, NC 27157; \\ ${ }^{2}$ FirmLogic LLC, Winston-Salem, NC 27101; ${ }^{3}$ Department of General Surgery (Surgical Oncology \\ Section), Wake Forest University School of Medicine, Winston-Salem, NC 27157, USA
}

Received August 8, 2007; Accepted September 28, 2007

\begin{abstract}
The goal of this study was to identify systematic alterations in key cell signaling and metabolic pathways that occur during colon cancer carcinogenesis and metastasis. Understanding gene expression changes in the context of specific pathways may increase our understanding of carcinogenesis and help guide treatment. Ten cases, with matched controls, were profiled for expression of $>18,000$ human transcripts using Affymetrix U133A chips. Data were filtered using GeneSifter ${ }^{\mathrm{TM}}$. Gene expression levels for primary colon samples were compared to a normal colon while metastatic tissues were compared to the primary colon. Differentially regulated genes were associated using the Kyoto encyclopedia of genes and genome pathways to identify cell signaling and metabolic pathways altered during carcinogenesis and metastasis. Primary colon samples displayed high positive $\mathrm{z}$-scores (indicating a gene ontology term that occurs more frequently than expected) for genes involved in Wnt-signaling (4.11), nitrogen metabolism (7.30) and inositol phosphate metabolism (2.47). Expression level changes for individual genes in each cluster were statistically significant (e.g. p=0.017 for cyclin D1 in the Wnt-signaling cluster). Metastatic tissue from the liver and omentum, but not the lung, displayed a decreased expression of genes important for oxidative phosphorylation. The metastatic tissue from all sites displayed a substantially decreased expression for genes involved in butanoate and propanoate metabolism and valine, leucine and isoleucine degradation. Our results demonstrate that systematic changes in gene expression occur for proteins involved in key cell signaling and metabolic pathways during the course of carcinogenesis and metastasis. These expression level changes
\end{abstract}

Correspondence to: Dr Perry Shen, Wake Forest University Baptist Medical Center, Medical Center Boulevard, Winston-Salem, NC 27157, USA

E-mail: pshen@wfubmc.edu

Key words: colorectal cancer, metastases, gene expression, molecular profiling complement the spectrum of mutations that characterize the progression of colorectal cancer.

\section{Introduction}

Colorectal cancer (CRC) is the second leading cause of cancerrelated death in the U.S., and nearly 150,000 new cases of $\mathrm{CRC}$ are reported annually. The genetic changes that cause colon cancer have been extensively studied and include early mutations in adenomatous polyposis coli (APC) and Ki-Ras and later mutations in p53 $(1,2)$. In addition to the mutations to these important oncogenes, colon cancer initiation and progression are accompanied by changes in gene expression. Several microarray studies have been undertaken to more fully elucidate the changes in gene expression that occur during CRC initiation, progression and metastasis (3-6).

In the present study, we report on the analysis of DNA microarray data obtained from primary colon tumors, as well as metastatic tissue from the liver, lung and omentum. A KEGG (Kyoto encyclopedia of genes and genomes) analysis of the gene expression data was performed to identify specific pathways that are either over- or under-represented in the array data (7). The KEGG analysis provides a depiction of how expression levels for groups of genes in a common pathway are altered in two different samples (e.g. primary colon tumor versus normal colon).

The results of the KEGG analysis implicated gene expression changes in several of the well-established pathways associated with CRC carcinogenesis (e.g. the Wnt-signaling pathway) (8). The KEGG analysis also revealed a systematic down-regulation of oxidative phosphorylation in the metastases from the liver and omentum consistent with an increased utilization of glycolysis even under aerobic conditions (i.e., the Warburg effect) at these metastatic sites. In addition, the current studies reveal systematic changes in other pathways (e.g. the coagulation cascade) that have yet to be established as having a significant role in CRC carcinogenesis or metastasis.

\section{Materials and methods}

Colorectal cancer patients and samples. A total of 20 samples (10 tumor samples and the corresponding normal tissue controls) were profiled using DNA microarrays. Tissue samples were obtained from 10 patients with sporadic colorectal 
Table I. Characteristics of the tumor tissues used in the gene expression analyses.

\begin{tabular}{lcccccc}
\hline ID number & Tumor site & Diagnostic site & Diagnostic group & Gender & Race & Age \\
\hline $1999-33$ & Liver & Colon & Adenocarcinoma & F & W & 64 \\
$1999-131$ & Liver & Colon & Adenocarcinoma & M & W & 45 \\
$2002-363$ & Liver & Colon & Adenocarcinoma & M & W & 65 \\
$2000-92$ & Omentum & Colon & Adenocarcinoma & F & W & 27 \\
$2000-215$ & Omentum & Colon & Adenocarcinoma & M & W & 64 \\
$2000-213$ & Lung & Colon & Adenocarcinoma & M & B & 49 \\
$2003-38$ & Lung & Colon & Adenocarcinoma & M & W & 75 \\
$2001-249$ & Colon & Colon & Adenocarcinoma & M & W & 44 \\
$2002-62$ & Colon & Colon & Adenocarcinoma & M & W & 59 \\
$2003-43$ & Rectum & Rectum & Adenocarcinoma & M & W & 64 \\
\hline
\end{tabular}

adenocarcinoma who underwent surgery at the Wake Forest University Comprehensive Cancer Center between 1999 and 2003. This study was approved by the Wake Forest University Baptist Medical Center Institutional Review Board. Tumor samples were staged after light microscopic visualization (Dr M. Willingham). All samples were classified as adenocarcinomas. The characteristics of the patients and tumors are listed in Table I. Non-tumor tissue was carefully removed from the tumor samples so that an estimated $80 \%$ of all tumor samples were comprised of tumor cells. Following macrodissection, all samples were frozen within $30 \mathrm{~min}$ of removal in liquid nitrogen for subsequent molecular analyses.

RNA isolation. Frozen tissue samples were pulverized with a mortar and pestle, and RNA was isolated using Trizol reagent (Invitrogen, Carlsbad, CA, USA). RNA concentration was measured by UV spectrophotometry at 260 and $280 \mathrm{~nm}$. RNA was further purified with RNAqueous columns (Ambion, Austin, TX, USA), and each sample was analyzed by electrophoresis on denaturing agarose gels and on an RNA 6000 Nano LabChip on the Agilent 2100 bioanalyzer (Agilent Technologies, Palo Alto, CA, USA) to ensure RNA integrity. The RNA samples were then stored at $-80^{\circ} \mathrm{C}$ until used for microarray hybridization experiments .

Affymetrix GeneChip hybridization. Ten micrograms of total RNA were used to synthesize double-stranded cDNA using SuperScript II reverse transcriptase (Invitrogen) and a T7(dT) 24 primer (Genset Corp., San Diego, CA). Biotinylated cRNA was synthesized from double-stranded cDNA using the Enzo Bioarray High Yield transcript labelling kit (Enzo Life Sciences, Farmingdale, NY), purified using the GeneChip sample cleanup module (Affymetrix, Santa Clara, CA) and fragmented. Fragmented cRNA was hybridized to Affymetrix HGU133 Plus 2.0 oligonucleotide arrays, washed, stained with streptavidin-phycoerythrin and analyzed.

Microarray data analysis and statistics. Normalized data from the Affymetrix MAS 5.0 software package were uploaded to GeneSifter ${ }^{\mathrm{TM}}$ (Www.genesifter.net; VizX Labs LLC, Seattle, WA, USA) for analysis. A differential gene expression analysis was performed by averaging the normalized (and log- transformed) samples and running a pair-wise comparison. Statistical significance was determined by the Student's t-test (two-tailed, unpaired). Genes with p-values $<0.05$ that were either up- or down-regulated more than 2-fold were included in the analysis. The KEGG reports, including z-score analyses, were generated by GeneSifter.

Scatterplot analysis. Scatterplot analyses were performed to evaluate the expression variability for samples from within the same group (i.e. normal versus normal colon) and for samples from different groups (i.e. normal colon versus primary colon tumor). Scatterplots revealed a higher intergroup variability relative to intra-group variability, as expected (data not shown).

\section{Results}

Systematic changes in the Wnt-signaling pathway in primary colon cancer. An analysis of the microarray data revealed 251 genes that were significantly up- or down-regulated in primary colon cancer relative to normal colon tissue $(p<0.05$, minimum 2-fold change). Genes were grouped into pathways using KEGG ontologies (7) to gain a more systematic understanding of how an altered expression affects important cellular processes, including cell-signaling and apoptosis. The number of genes that were either up- or down-regulated in the primary colon tissue relative to normal colon were then evaluated in each pathway.

The primary colon tumor samples displayed a significantly altered expression for genes involved in the Wnt-signaling pathway (Table II). The Wnt-signaling pathway plays an important role in regulating proliferation in the colonic epithelium (9). More than $70 \%$ of colorectal cancers are defective in some aspect of the Wnt-signaling pathway (10). Mutation in APC, an event not detectable by gene expression profiling, is frequently among the earliest events in CRC etiology (1). Overall, 4 out of the 126 genes included in the Wnt-signaling pathway cluster were significantly up-regulated in the primary colon tumor relative to normal colon tissue. The z-score for the Wnt-signaling pathway for the primary colon tumor relative to normal colon tissue was 4.11 . Specific changes in gene expression for proteins involved 
Table II. Differentially regulated genes by pathway: colon primary tumors versus colon normal tissue

\begin{tabular}{|c|c|c|c|c|c|}
\hline Pathway & Gene name & $\begin{array}{c}\text { Fold } \\
\text { change }\end{array}$ & $\begin{array}{l}\text { Direction } \\
\text { of change }\end{array}$ & p-value & Gene ID \\
\hline \multirow[t]{4}{*}{ Wnt signaling } & $\begin{array}{l}\text { Cyclin D1 (PRAD1: parathyroid } \\
\text { adenomatosis 1) }\end{array}$ & 2.33 & Up & 0.017 & BC000076 \\
\hline & $\begin{array}{l}\text { Matrix metalloproteinase } 7 \\
\text { (matrilysin, uterine) }\end{array}$ & 6.25 & Up & 0.008 & NM_002423 \\
\hline & Phospholipase C, ß 4 & 6.07 & Up & 0.035 & NM_000933 \\
\hline & $\begin{array}{l}\text { V-myc myelocytomatosis viral } \\
\text { oncogene homolog (avian) }\end{array}$ & 2.23 & Up & 0.025 & NM_002467 \\
\hline \multirow[t]{4}{*}{ Nitrogen metabolism } & Carbonic anhydrase I & 32.96 & Down & 0.014 & NM_001738 \\
\hline & Carbonic anhydrase II & 5.12 & Down & 0.004 & M36532 \\
\hline & Carbonic anhydrase IV & 18.93 & Down & 0.041 & NM_000717 \\
\hline & Carbonic anhydrase XII & 4.49 & Down & 0.034 & NM_001218 \\
\hline \multirow[t]{4}{*}{ Inositol phosphate metabolism } & $\begin{array}{l}\text { Dual-specificity tyrosine-(Y)- } \\
\text { phosphorylation regulated kinase } 2\end{array}$ & 2.24 & Down & 0.025 & Y09216 \\
\hline & G protein-coupled receptor kinase 5 & 3.12 & Down & 0.032 & NM_005308 \\
\hline & Phospholipase C, $ß 4$ & 6.07 & Up & 0.035 & NM_000933 \\
\hline & Phospholipase C, $\varepsilon 1$ & 2.42 & Down & 0.015 & NM_016341 \\
\hline
\end{tabular}

in the Wnt-signaling pathway included a 2.3 -fold up-regulation of cyclin D1 ( $\mathrm{p}=0.017)$, a 2.2-fold up-regulation of $\mathrm{v}$-myc $(\mathrm{p}=0.025)$ and $>6$-fold increases in MMP7 and phospholipase $\mathrm{C}$ (PLC- 34 ) expression ( $\mathrm{p}=0.025$ and 0.035 , respectively, Table II). Both cyclin D and myc enhance cellular proliferation, while MMPs, including MMP7, are important for tumor invasiveness. Our data demonstrate that an elevated expression of proteins that enhances cellular proliferation as a result of Wnt-signaling, such as cyclin-D and myc, plays a significant role in CRC carcinogenesis. These changes in gene expression are complementary to the APC mutations for establishing the tumor phenotype. Proteins that enhance invasiveness as a result of Wnt-signaling, such as MMP7, also display an elevated gene expression in the primary colon tumors relative to the normal colon cells.

Other pathways altered in primary colon cancer. In addition to the altered expression for genes involved in the Wntsignaling pathway, the expression of genes involved in nitrogen (Table II) and inositol phosphate metabolism (Table II), were altered in the primary colon tumor relative to the expression levels in normal colon samples. The z-score for the nitrogen metabolism cluster (colon primary versus normal colon) was 7.30, while the $\mathrm{z}$-score for inositol phosphate metabolism was 2.47 . In the present study four isoforms of carbonic anhydrase (CA) were observed to have decreased expression in colon tumor tissue relative to normal colon (Table II). Carbonic anhydrase (CA) catalyzes the hydration of carbon dioxide that is important for nitrogen metabolism. CA activity is important for anion and fluid balance in cells. CA isoforms have a decreased expression in and include CA that is membrane-bound (CA IV and XII) and CA that is cytosolic (CA I and II). Elevated levels of CA XII have previously been shown to be associated with tumor hypoxia (11). Changes in the inositol phosphate metabolism included an elevated expression of PLC B4 and a decreased expression of PLC $\varepsilon 1$. An altered expression of PLC and other genes involved in inositol phosphate metabolism reflects changes in the cell-signaling pathways important for primary colon cancer initiation and progression (Fig. 1).

Tissue-specific gene expression changes in metastatic CRC. An analysis of gene expression changes in the metastases to the liver, lung and omentum revealed a substantial variability in the number of genes undergoing significant expression level changes at each metastatic site. In addition, relatively few genes displayed common expression level changes for two or more metastatic sites (Fig. 2). The greatest number of genes undergoing expression level changes of at least 2-fold relative to the primary colon tumors occurred for liver metastases, $n=505$. Of these 505 genes, 387 were unique to the liver and did not occur for either the lung or omentum metastases. The fewest number of genes undergoing significant expression level changes occurred in the lung $(n=228)$ and 142 of these genes were unique to lung metastases. Genes $(n=369)$ were significantly altered in the metastases to the omentum with 257 of these occurring only in the metastases to the omentum. The expression levels of only 28 genes were significantly altered in the metastases at all three sites relative to the normal colon (Fig. 2). The number of genes undergoing significant expression level differences at the three metastatic sites relative to the primary colon is similar to the 251 genes that displayed an altered 


\title{
$\mathbf{N}=\mathbf{2 5 1}$ \\ Normal Colon \\ Wnt-signaling \\ Nitrogen Metabolism \\ Inositol-phosphate Metabolism
}

\author{
$\mathrm{N}=369 ; \mathrm{U}=257$ Omentum Metastases \\ Oxidative Phosphorylation \\ V,L,I Degradation \\ Coagulation \& Complement Cascade \\ Primary Colon \\ $\mathrm{N}=505 ; \mathrm{U}=\mathbf{3 8 7}$ Liver Metastases \\ Oxidative Phosphorylation \\ V,L,I Degradation \\ Coagulation \& Complement Cascade \\ $N=228 ; U=142$ \\ V,L,I Degradation
}

Figure 1. The number of genes $(\mathrm{N})$ and the number of unique genes $(\mathrm{U})$ that significantly change the expression during the progression of the normal to the primary colon tumor (top) and during the progression of the primary colon to the liver, lung, or omentum metastases (bottom). Processes that undergo concerted change as a result of altered gene expression, e.g. Wnt-signaling in the case of normal to primary colon tumor progression, are indicated for each transformation.

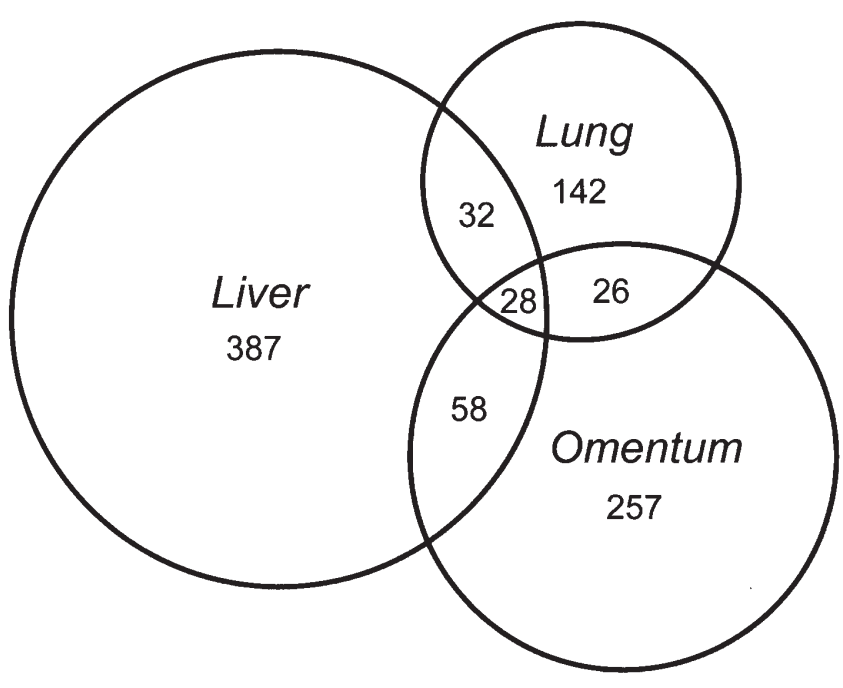

Figure 2. A Venn diagram depicting the numbers of genes that are differentially expressed in colon cancer metastases to the liver, lung and omentum. The numbers in the overlapping circles indicate the number of genes differentially expressed in two or three tumor sites. A 2-fold change in gene expression was considered as significant.

expression in the primary colon tumor relative to the normal colon tissue. The metastatic tissue displayed a much greater variability relative to the normal tissue from the same site, as expected (e.g. 1408 genes displayed significant expression changes in the liver metastases relative to the normal liver).

Systematic changes in the oxidative phosphorylation pathway. Most tumor cells have an enhanced reliance on anaerobic glycolysis, a phenomenon known as the 'Warburg effect' (12). It was proposed that the metabolism of glucose to lactate by tumor cells, even under aerobic conditions, results from selective pressure during periods of hypoxia over the course of tumor development. The gene expression profile responsible for the switch to anaerobic glycolysis in tumor cells, however, remains largely uncharacterized. Recently, it was shown that $\mathrm{p} 53$ regulates the expression of cytochrome 2 oxidase ( $\mathrm{SCO} 2$ ) synthesis and that a disruption of the $\mathrm{SCO} 2$ expression can cause a shift to glycolysis (13). A mutated transketolase transcript (TKTLI) has also been shown to be expressed at high levels in the invasive colon and urothelial tumors (14). Transketolase enzymes control the non-oxidative portion of the pentose phosphate pathway important for glucose conversion to lactate. Thus, the gene profile signature for anaerobic glycolysis may be largely undetectable by gene array analysis.

In the present study, the oxidative phosphorylation pathway is very strongly down-regulated in the liver metastases $(z=-6.05)$ and strongly down-regulated in the omentum metastases $(z=-3.96)$ relative to the primary colon tumor tissue. Sixteen proteins involved in the oxidative phosphorylation pathway were down-regulated in the liver metastases relative to the primary colon. Eight proteins involved in oxidative phosphorylation were down-regulated in the omentum metastases relative to the primary colon. Among the proteins that were down-regulated in the liver metastases relative to the normal colon tissue were cytotchrome c oxidase subunits $\mathrm{Vb}$ and VIIb. These data are consistent with cytotchrome $\mathrm{c}$ oxidase playing a central role in the metabolic switch of cancer cells to glycolysis. A list of the genes involved in the oxidative phosphorylation pathway that are down-regulated in the liver metastases is included in Table III. Although not included in the oxidative phosphorylation pathway used in the KEGG analysis, transketolase transcripts were also significantly up- 
Table III. Oxidative phosphorylation-associated genes down-regulated in the liver metastatic tumors relative to the primary colon adenocarcinomas.

ATP synthase, $\mathrm{H}+$ transporting, mitochondrial F0 complex, subunit c (subunit 9)

ATPase, $\mathrm{H}+$ transporting, lysosomal $38 \mathrm{kDa}$, V0 subunit $\mathrm{d}$ isoform 1

ATPase, $\mathrm{H}+$ transporting, lysosomal $70 \mathrm{kDa}, \mathrm{V} 1$ subunit A

Cytochrome c oxidase subunit $\mathrm{Vb}$

Cytochrome c oxidase subunit VIIb

NADH dehydrogenase (ubiquinone) $1 \alpha$ subcomplex, 10,42 kDa

NADH dehydrogenase (ubiquinone) $1 \alpha$ subcomplex, 13

NADH dehydrogenase (ubiquinone) $1 \alpha$ subcomplex, 8, $19 \mathrm{kDa}$

NADH dehydrogenase (ubiquinone) Fe-S protein 1, $75 \mathrm{kDa}$ (NADH-coenzyme Q reductase)

NADH dehydrogenase (ubiquinone) Fe-S protein 3, $30 \mathrm{kDa}$ (NADH-coenzyme Q reductase)

NADH dehydrogenase (ubiquinone) Fe-S protein 5, $15 \mathrm{kDa}$ (NADH-coenzyme $\mathrm{Q}$ reductase)

NADH dehydrogenase (ubiquinone) Fe-S protein 6,13 kDa (NADH-coenzyme Q reductase)

NADH dehydrogenase (ubiquinone) Fe-S protein 7, $20 \mathrm{kDa}$ (NADH-coenzyme Q reductase)

NADH dehydrogenase (ubiquinone) Fe-S protein 8, $23 \mathrm{kDa}$ (NADH-coenzyme Q reductase)

NADH dehydrogenase (ubiquinone) flavoprotein $1,51 \mathrm{kDa}$

Ubiquinol-cytochrome c reductase core protein I

Table IV. Z-scores for select metabolic pathways in metastatic tissues relative to the primary colon adenocarcinoma.

\begin{tabular}{lccc}
\hline Pathway & Liver & $\begin{array}{c}\text { Metastatic tumor site } \\
\text { Lung }\end{array}$ & Omentum \\
\hline Butanoate metabolism & 3.56 & 3.48 & 8.87 \\
Propanoate metabolism & 3.54 & 4.22 & 7.98 \\
Valine, leucine, isoleucine degradation & 3.56 & 4.88 & 7.78 \\
\hline
\end{tabular}

regulated in the liver metastases relative to the normal liver (ratio $=3.76, \mathrm{p}=0.002$ ).

Other metabolic changes in metastatic CRC. Gene expression profiles for metastatic tissues from the liver, lung and omentum were compared to the primary colon tumor tissue to identify tissue-specific and tissue-independent changes in gene expression. Gene expression levels for the three metastatic sites all displayed a significantly reduced expression of genes involved in butanoate metabolism and propanoate metabolism and valine, leucine and isoleucine degradation relative to normal colon tissue. The $\mathrm{z}$-scores for these pathways in the liver, lung and omentum metastatic tissues relative to primary colon are shown in Table IV. In all cases, the genes in these metabolic pathways occurred less frequently in the metastatic tissues compared to the corresponding primary colon tumor. The genes included in these pathways include acetoacetyl-CoA synthetase, aldehyde dehydrogenases (H3A2, H7A1) and aldose reductases. For example, L-3-hydroxyacyl-coenzyme A dehydrogenase (short chain), a gene involved in both butanoate metabolism and valine, leucine and isoleucine degradation was down-regulated in tissue from all three metastatic sites relative to the primary colon tumor tissue with $\mathrm{p}=0.024$ (liver), $\mathrm{p}=0.022$ (lung) and $\mathrm{p}=0.005$ (omentum).
The z-scores for valine, leucine and isoleucine degradation for all metastatic sites demonstrated a significant reduction in the expression of several genes important for this pathway in metastatic tissue. This result is somewhat surprising in light of reports that elevated branched-chain amino acid metabolism is characteristic of cancer cells, and that dietary supplementation of BCAA may reduce weight-loss experienced by cancer patients $(15,16)$.

Expression profiles for coagulation and the complement cascade. Coagulation factors are known to play an important role in tumor metastasis. The secretion of fibrinogen by tumor cells may facilitate the formation of clots that may be important for metastasis formation (17). Alternatively, tumor cells may secrete VEGF, which in turn causes fibroblasts to secrete fibronectin to facilitate formation of pre-metastatic niches (18). Fibrinogen secretion by tumor cells may also reduce the function of natural killer (NK) cells and thus help tumor cells evade immune surveillance (19). The gene expression profile for metastatic tissue with respect to the blood coagulation pathway has not been characterized. In the present study, the z-value for the coagulation and complement cascade pathway for liver metastases relative to primary colon samples was 8.08 while that for omentum metastases was 3.99. Specific genes in the blood coagulation pathway that 
were up-regulated in liver metastases included the coagulation factor II (thrombin) as well as fibrinogen the $\beta$ and $\gamma$ chains. Thus, the data are consistent with the up-regulation of the expression of blood coagulation factors by tumor cells, or associated stromal cells, contributing to metastasis formation from the primary colon tumor cells to the liver and omentum.

\section{Discussion}

The initiation of colon cancer and subsequent development of metastases at the liver, lung and in the omentum are complex processes that proceed with changes in gene expression as well as with mutations and changes to the extracellular matrix. The present study focused on identifying gene expression changes that occur in primary colon tumor tissue relative to normal colonic epithelial tissue, as well as gene expression changes in metastatic tissue from the lung, liver and omentum relative to the primary colon tumor. KEGG analyses were performed to evaluate the impact of gene expression changes to metabolic and cell-signaling pathways. The use of KEGG analyses renders the observed results less dependent on the expression level changes for a specific gene since the expression in the contextual environment of a particular pathway is taken into account.

Our results are consistent with colon cancer carcinogenesis involving an altered expression of genes important for cellsignaling and especially for the Wnt-signaling pathway. While previous studies reported the importance of deletions and other mutations to APC and other genes in the Wntsignaling pathway for colon cancer carcinogenesis $(1,2)$, the present results are the first to use gene array technology and KEGG analysis to establish that gene expression changes are also significant in the activation of the Wnt-signaling pathway during colon cancer carcinogenesis.

The gene expression data for the oxidative phosphorylation pathway are also among the first to describe gene expression changes that accompany a metabolic switch in cancer cells to glycolysis in a systematic manner. The down-regulation of the oxidative phosphorylation pathway was clearly evident for the liver metastases and to a lesser extent the omentum metastases, relative to primary colon tissue. Notably, the down-regulation of the oxidative phosphorylation pathway was not apparent for lung metastases relative to primary colon tumor tissue. If down-regulation of oxidative phosphorylation occurs in response to episodic hypoxia, lung metastases, which are likely to be less susceptible to hypoxia than metastases to other organs, would be less likely than metastases to other sites to down-regulate the oxidative phosphorylation pathway. It is not clear if the lack of significant changes in gene expression for the oxidative phosphorylation pathway in primary colon tissue relative to normal colon tissue results from the primary tumor not primarily using glycolysis, or if alternative mechanisms are operative in primary colon cancer.

Our results are consistent with fundamental changes in the expression of genes important for basic metabolism accompanying the transformation from primary tumor to a metastatic lesion, regardless of the metastatic site. The data reveal that selective pressure during tumor development and especially during metastasis, alters diverse processes of cellular metabolism. Previous array studies demonstrated the upregulation of aldehyde dehydrogenase and aldo-keto reductase in malignant ascites relative to the primary tumor (20). The up-regulation of these enzymes in metastatic tissue was attributed to a role in drug metabolism. For example, the sensitivity of breast cancer cells to cyclophosphamide was shown to be inversely correlated to the expression levels for ALDH1A1 and ALDH3A1 (21). In the present study, ALDH3A2 and ALDH7A1 were observed to be downregulated in metastatic tissue relative to primary colon tumor tissue. A previous study demonstrated that three variants of ALDH3 were up-regulated in hepatocellular carcinoma while two variants of ALDH2 became undetectable in these malignant cells (22). Thus, the pattern of ALDH expression may be signatory to specific malignancies.

The present study demonstrated that cancer cells from both primary tumor and metastatic sites have altered the expression of genes involved in amino acid metabolism and other basic metabolic processes, and that the expression of these genes can be tissue- or independent-specific. These results are consistent with the recent publication from Sjoblom et al which reported a detailed analysis of the human genome sequence in 11 colorectal cancers and found the vast majority of mutated genes were determined as playing an important role in cellular function (23). The present data confirm that the gene expression changes detected in the present study are logically organized into pathways that are consistent with what has previously been reported for colon cancer initiation and progression.

\section{Acknowledgements}

This work was supported by NIH-NCI P30 CA12197 and the Comprehensive Cancer Center of Wake Forest University. The authors are grateful to Dr Mark Willingham for the staging of the tumor samples, Dr Yong Chen for assistance with the microarray analysis, Mr. Russell Norris and Mr. Lance Warren for performing RNA extraction procedures, Dr Steven Walker for assistance with GeneSifter, and to the CCCWFU for financial support.

\section{References}

1. Kinzler KW and Vogelstein B: Lessons from hereditary colorectal cancer. Cell 87: 159-170, 1996.

2. de la Chappelle A: Genetic predisposition to colorectal cancer. Nat Rev Cancer 4: 769-780, 2004.

3. Hegde P, Qi R, Gaspard R, et al: Identification of tumor markers in models of human colorectal cancer using a 19,200-element complementary DNA microarray. Cancer Res 61: 7792-7797, 2001 .

4. Yanagawa R, Furukawa Y, Tsunoda T, et al: Genome-wide screening of genes showing altered expression in liver metastases of human colorectal cancers by cDNA microarray. Neoplasia 3: 395-401, 2001.

5. Koehler A, Bataille F, Schmid C, et al: Gene expression profiling of colorectal cancer and metastases divides tumors according to their clinicopathological stage. J Pathol 204: 65-74, 2004.

6. Li M, Lin YM, Hasegawa S, et al: Genes associated with liver metastasis of colon cancer, identified by genome-wide cDNA microarray. Int J Oncol 24: 305-312, 2004.

7. Green ML and Karp PD: The outcomes of pathway database computations depend on pathway ontology. Nucleic Acids Res 34: 3687-3697, 2006.

8. Bienz $\mathrm{M}$ and Clevers H: Linking colorectal cancer to Wntsignaling. Cell 103: 311-320, 2000.

9. Radtke F and Clevers H: Self-renewal and cancer of the gut: two sides of a coin. Science 307: 1904-1909, 2005 
10. Kolligs FT, Bommer G and Goke B: Wnt/B-catenin/Tef Signaling: A critical pathway in gastrointestinal tumorigenesis. Digestion 66: 131-144, 2002.

11. Le QT, Chen E, Salim A, et al: An evaluation of tumor oxygenation and gene expression in patients with early stage nonsmall cell lung cancers. Clin Cancer Res 12: 1507-1514, 2006.

12. Gatenby RA and Gillies RJ: Why do cancers have high aerobic glycolysis? Nat Rev Cancer 4: 891-899, 2004.

13. Matoba S, Kang JG, Patino WD, et al: P53 regulates mitochondrial respiration. Science 312: 1650-1653, 2006.

14. Langbein S, Zerilli M, Zur Hausen A, et al: Expression of transketolase TKTLI predicts colon and urotheliel cancer patient survival: Warburg effect reinterpreted. Br J Cancer 94: 578-585, 2006.

15. Siddiqui R, Pandya D, Harvey K and Zaloga GP: Nutrition modulation of cachexia/proteolysis. Nutr Clin Pract 21: 155-167, 2006.

16. Choudry HA, Pan M, Karinch AM and Souba WW: Branchedchain amino acid-enriched nutritional support in surgical and cancer patients. J Nutr 136: 14S-18S, 2006.

17. Im JH, Fu W, Wang H, Bhatia SK, Hammer DA, Kowalska MA and Muschel RJ: Coagulation facilitates tumor cell spreading in the pulmonary vasculature during early metastatic colony formation. Cancer Res 64: 8613-8619, 2004.

18. Kaplan RN, Riba RD, Zacharoulis S, et al: VEGFR1-positive haematopoietic bone marrow progenitors initiate the premetastatic niche. Nature 438: 820-827, 2005.
19. Palumbo JS, Talmage KE, Massari JV, et al: Platelets and fibrin(ogen) increase metastatic potential by impeding natural killer cell-mediated elimination of tumor cells. Blood 105: 178-185, 2005.

20. Sakakura C, Hagiwara A, Nakanishi M, et al: Differential gene expression profiles of gastric cancer cells established from primary tumor and malignant ascites. Br J Cancer 87: 1153-1161, 2002.

21. Sreerama L and Sladek NE: Primary breast tumor levels of suspected molecular determinants of cellular sensitivity to cyclophosphamide, ifosfamide and certain other anticancer agents as predictors of paired metastatic tumor levels of these determinants. Rational individualization of cancer chemotherapeutic regimens. Cancer Chemother Pharmacol 47: 255-262, 2001.

22. Park KS, Cho SY and Paik YK: Proteomic alterations of the variants of human aldehyde dehydrogenase iosozymes correlate with hepaocellular carcinoma. Int J Cancer 97: 261-265, 2002.

23. Sjoblom T, Jones S, Wood LD, et al: The consensus coding sequences of human breast and colorectal cancers. Science 314: 268-274, 2006. 